\title{
Uniportal video assisted thoracic surgery right main sparing lung bronchial resection: live surgery in Sao Paulo
}

\author{
Rodrigo A. S. Sardenberg ${ }^{1}$, Diego Gonzalez-Rivas ${ }^{2,3}$ \\ ${ }^{1}$ Department of Thoracic Surgery, Hospital Alemão Oswaldo Cruz, São Paulo, Brazil; ${ }^{2}$ Department of Thoracic Surgery and Minimally Invasive \\ Thoracic Surgery Unit (UCTMI), Coruña University Hospital, Coruña, Spain; ${ }^{3}$ Department of Thoracic Surgery, Shanghai Pulmonary Hospital, \\ Tongji University School of Medicine, Shanghai 200433, China \\ Correspondence to: Rodrigo A.S. Sardenberg, MD. Department of Thoracic Surgery, Hospital Alemão Oswaldo Cruz, Rodrigo Claudio street, 477 ap \\ 61, São Paulo, Brazil. Email: rodafs@uol.com.br.
}

\begin{abstract}
We report the first uniportal right main sparing bronchial resection in Brazil. The procedure was performed live at Hospital Alemão Oswaldo Cruz, São Paulo during the II Brazil uniportal video assisted thoracic surgery (VATS) advanced masterclass. Another uniportal VATS right upper lobectomy with bronchoplasty, uniportal VATS S6 left segmentectomy and a subxiphoid uniportal VATS mediastinal tumor, were performed as well. This VATS program was conducted in São Paulo for the first time in 2016. We hope to extend the course to other centers around the country in the next year.
\end{abstract}

Keywords: Uniportal video assisted thoracic surgery (Uniportal VATS); bronchoplasty; carcinoid tumor

Received: 15 October 2017; Accepted: 18 October 2017; Published: 11 November 2017.

doi: 10.21037 /jovs.2017.10.14

View this article at: http://dx.doi.org/10.21037/jovs.2017.10.14

\section{Introduction}

Thoracoscopic lobectomies have been performed since 1992 (1), following the worldwide acceptance of this new approach. Although being available for at least 20 years worldwide, has not become the universal approach yet, since most lobectomies are still performed by open approach. Complex pulmonary resections, such as our case, have been gained more field on video assisted thoracic surgery (VATS) surgeons. Overtime, it has been clear the benefits for the patients from minimally invasive approach, without oncologic compromising. The uniportal VATS is a new technique with convincing results for early stage and advanced tumors (2). Progress has been made since the first simple lobectomies to the more complex procedures.

Better and new thoracoscopic instrumentation, growing technology, knowledge worldwide spread, and acceptable oncologic results, contributed to its evolution (3).

Carcinoid tumors are neuroendocrine lung tumors that occur in $1-2 \%$ of all lung cancers, with a good prognosis when complete surgical excision is performed (4). Due its endoluminal location, they are characterized by causing respiratory symptoms such as cough, hemoptysis, recurrent respiratory infections, and asthma simulation.

Lung carcinoid tumors contributes to $1-2 \%$ of all lung cancers, with a good prognosis when complete resection is performed (4). They have been reported since 1947 , and the term "bronchoplasty" was concepted in 1955 (4). Because the high endoluminal location incidence, they can cause respiratory symptoms such as dry cough, recurrent respiratory infections, and asthma simulation.

For the last 10 years, different techniques of minimally invasive surgery for bronchial resections have been introduced, including the uniportal VATS surgery $(3,5)$.

We report a case in which a uniportal VATS right main sparing lung bronchial resection was performed live, with excellent result.

\section{Patient selection}

A 77-year-old female patient with no morbidity or previous smoking history, presented with dyspnea and dry cough. The computed tomography showed a tumor on right main 


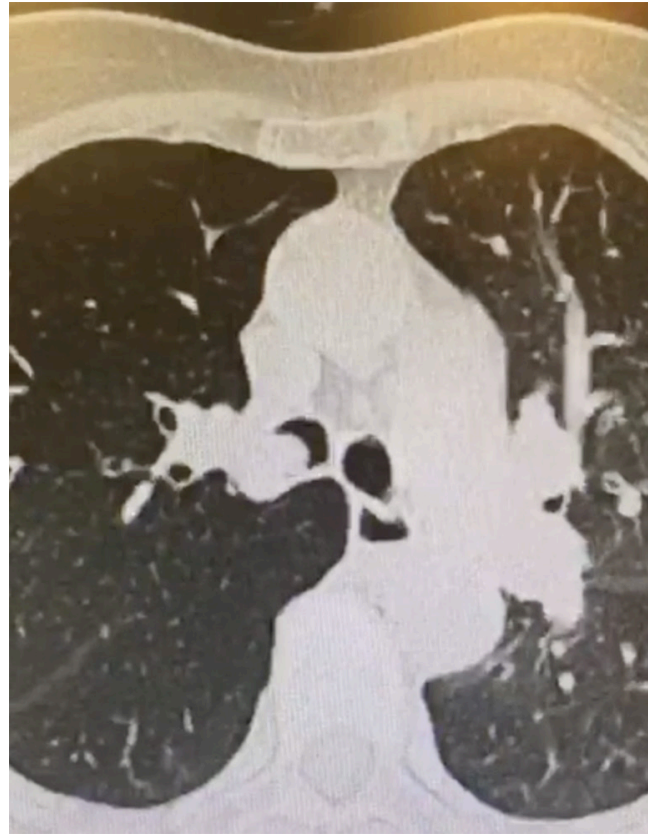

Figure 1 CT scan showing tumor on right main bronchus.

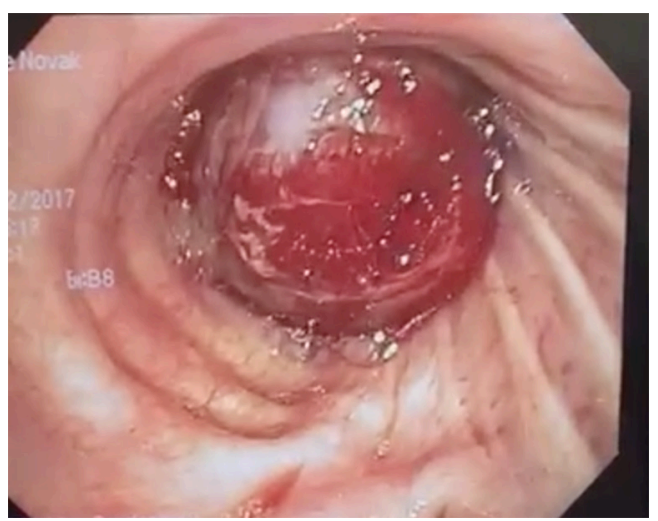

Figure 2 Bronchoscopic view of tumor on right main bronchus.

bronchus (RMB) (Figure 1). The bronchoscopy confirmed an endobronchial lesion in the right upper lobe bronchus (RULB) obstructing the RMB $2 \mathrm{~cm}$ below the carina (Figure 2). Biopsy confirmed typical carcinoid tumor. We considered this case for uniportal VATS during a live surgery event.

\section{Equipment}

A 30 degree Karl Storz thoracoscopic camera system was used. Thoracoscopic instruments were provided by Scanlan, and Endo-Gia staplers by Medtronic.

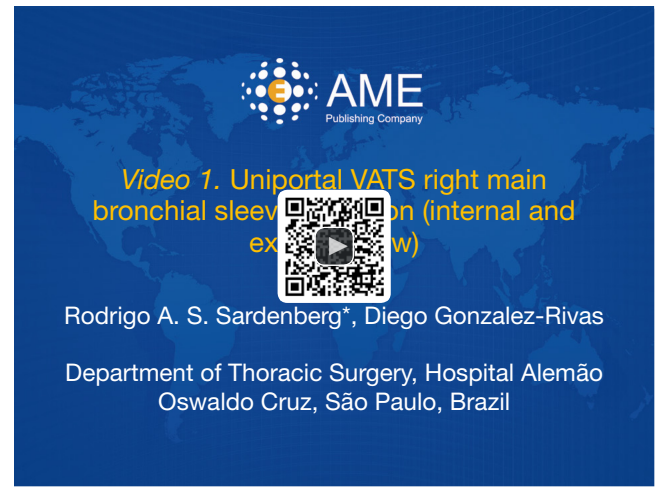

Figure 3 Uniportal VATS right main bronchial sleeve resection (internal and external view) (6). VATS, video assisted thoracic surgery. Available online: http://www.asvide.com/articles/1819

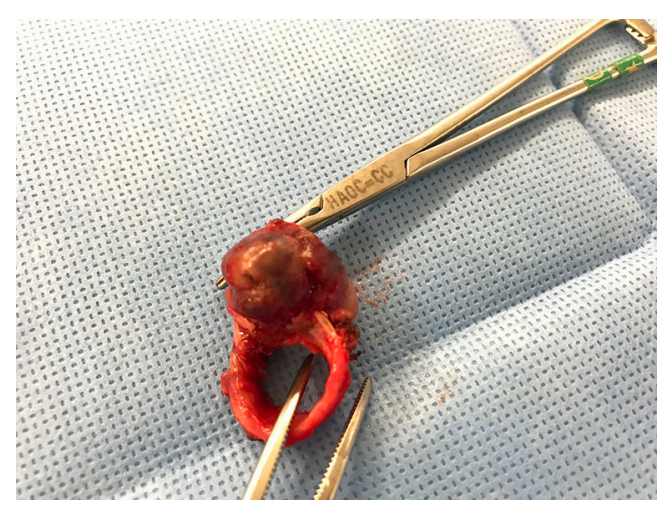

Figure 4 Bronchial segment with the tumor.

\section{Surgical procedure (Figure 3)}

The patient was placed in a left lateral decubitus position. A 4-cm incision was made in the fourth intercostal space and blunt dissection allowed access to the pleural space. The camera was placed posteriorly and the thoracoscopic instruments anteriorly. Inside the chest cavity, we realized the lung did not collapsed-since the tumor blocked the right main bronchus (RMB). It was necessary to perform a incision on the RMB $1 \mathrm{~cm}$ below the carina, and empty the whole lung prior to initiate the dissection. Once the lung collapsed, the azygos vein was stapled in order to better exposure of the trachea, carina and proximal aspect of the RMB. The dissection continued distally until the right upper and intermedius bronchi. An incision was made in the base of the right upper bronchus and a circumferential cut was made with scissors to the intermedius bronchus. The bronchus segment with the tumor was removed, with tumor 


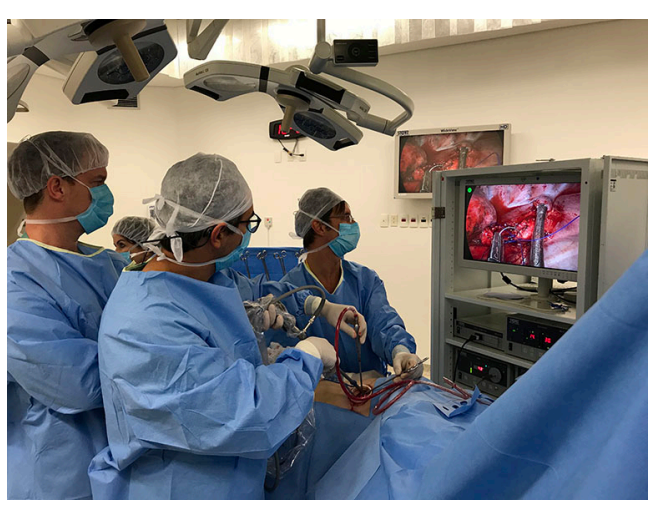

Figure 5 Photo showing surgical instrumentation during the anastomosis.

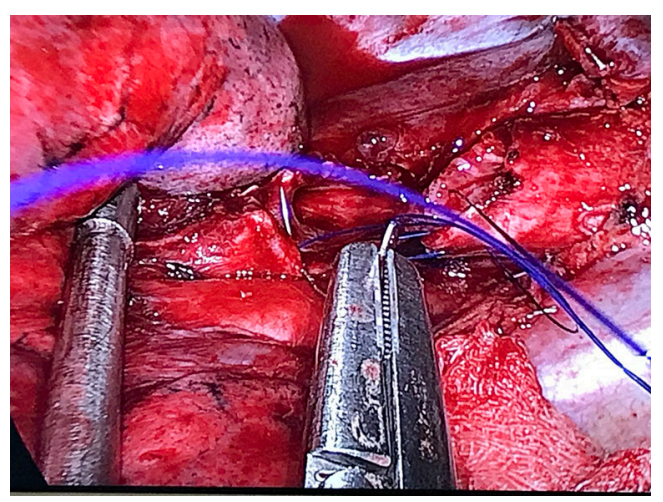

Figure 6 End-to-end anastomosis (RMB and ULB and IB). RMB, right main bronchus (RMB); ULB, upper lobe bronchus; IB, intermedius bronchus.

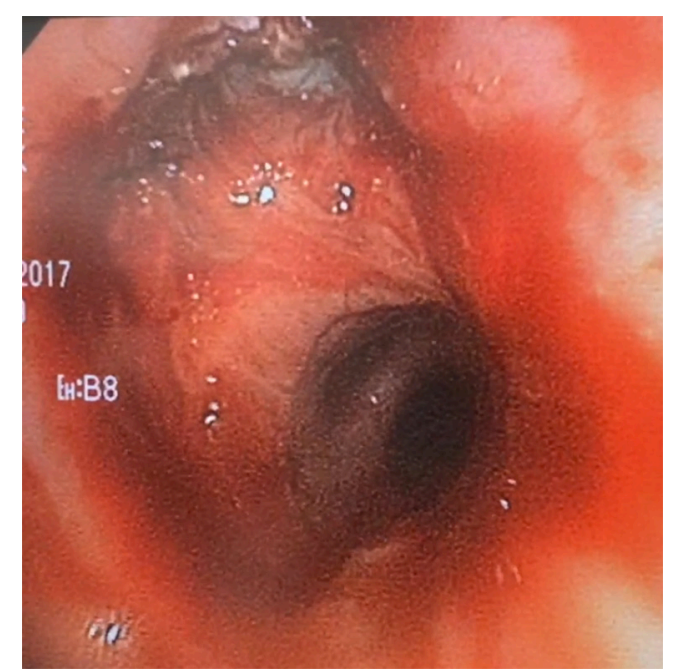

Figure 7 Post-operative bronchoscopic view of the anastomosis. located to the bronchial base (Figure 4). Frozen section confirmed carcinoid tumor with free margins, proximal and distally.

A complete incision with scissors in the right upper and the intermedius bronchus was performed, and the bronchus segment with the tumor was removed (Figure 4). Frozen section confirmed carcinoid tumor with free margins, proximal and distally.

Dissection continued until the exposure of the divided $\mathrm{RMB}$, and complete lymphadenectomy was necessary to facilitate bronchial anastomosis.

An end-to-end anastomosis with prolene $3 / 0$ was performed (1 thread with 2 needles) (Figure 5), starting with a posterior stitch in the cartilaginous-membranous junction to help approximate the right upper and intermedius bronchi to main stem bronchus (Figure 6). Once this lateral wall was completed, we used the other needle to perform a running suture in the superior membranous portion first and then we continued with the superior cartilaginous portion from posterior to anterior. The first running suture suture and the last suture were both tyed at the anterior portion of the bronchial circumference the with the help of a knot pusher. A test with water discarded air leak. No tissue flap was used to protect the anastomosis, and a single chest tube $28 \mathrm{~F}$ was placed through the incision. Post-operative bronchoscopy showed the anastomosis in good condition (Figure 7).

\section{Post-operative management}

The patient was kept in intensive care unit for $24 \mathrm{hs}$, and as soon she awaked, started mobilization and respiratory physiotherapy. No opioids were necessary for analgesia, and the patient was discharged on the third day.

\section{Discussion}

The uniportal VATS program started in 2016 with the visit of Dr. Gonzalez-Rivas in São Paulo. At that time, two upper lobectomies-right and left-were performed live successfully at the same day. During this course, 20 Brazilians surgeons were exposed to uniportal VATS techniques. This year, there were 3-day course-1-day theory on advanced uniportal techniques and 2 days live surgery. On the first surgery day, Dr. Gonzalez-Rivas performed a right upper lobectomy with bronchoplasty and a S6 left segmentectomy. On the second surgery day, Dr. GonzalezRivas performed the present case-right main sparing lung 
bronchial resection-and a mediastinal tumor resection through a subxiphoid access. All patients were discharged from the hospital on the third postoperative day without any complications.

In the beginning of this approach, surgeons normally perform a longer incision, and it gradually become smaller, until 3-4 $\mathrm{cm}$ in length.

As others VATS approaches, surgeons should be aware he has to open the chest, especially in the beginning of the experience. For more skilled and experienced surgeons, such as Dr. Gonzalez-Rivas, the complex proceduresbronchial and vascular sleeves-are possibly carried out by uniportal VATS as well.

Regarding uniportal VATS bronchial sutures, it can be performed with interrupted stitches, although it is trickier and takes more time. The usage of continuous and monofilament suture makes the thread movement easier, as well as the tying, and all knots preferably placed externally.

The bronchial edge must be assessed during the operation, the suture should check at the end of the surgery using insufflation under water and bronchoscopic control to check integrity and secretion aspiration.

Uniportal VATS has stimulated the new and more experienced surgeons to apply this technique, especially for early stage lung cancers. As new instruments, such as staplers and clips are emerging, thoracoscopic surgery has gained more adepts.

\section{Results}

As Brazil's development moves forward, the minimally invasive thoracic surgery in the country should follow this development. Unfortunately, most of Brazilians thoracic surgeons could not access this new and evolving technique, and the aim of this annual course is to reach as much surgeons we can over the country.

doi: 10.21037/jovs.2017.10.14

Cite this article as: Sardenberg RA, Gonzalez-Rivas D. Uniportal video assisted thoracic surgery right main sparing lung bronchial resection: live surgery in Sao Paulo. J Vis Surg 2017;3:166.

\section{Acknowledgements}

This course was supported by Medtronic.

\section{Footnote}

Conflicts of Interest: The authors have no conflicts of interest to declare.

Informed Consent: Written informed consent was obtained from the patient for publication of this manuscript and any accompanying images.

\section{References}

1. Roviaro G, Rebuffat C, Varoli F, et al. Videoendoscopic pulmonary lobectomy for cancer. Surg Laparosc Endosc 1992;2:244-7.

2. Gonzalez-Rivas D, Fernandez R, Fieira E, et al. Uniportal video-assisted thoracoscopic bronchial sleeve lobectomy: first report. J Thorac Cardiovasc Surg 2013;145:1676-7.

3. Gonzalez-Rivas D, Yang Y, Stupnik T, et al. Uniportal videoassisted thoracoscopic bronchovascular, tracheal and carinal resections. Eur J Cardiothorac Surg 2016;49 Suppl 1:i6-16.

4. Tsuchiya R. Bronchoplastic techniques. In: Pearson FG, Cooper JD, Deslauriers J, et al. editors. Thoracic Surgery. New York: Churchill Livingstone, 2002:1005-13.

5. Ng CS, Gonzalez-Rivas D, D'Amico TA, et al. Uniportal VATS-a new era in lung cancer surgery. J Thorac Dis 2015;7:1489-91.

6. Sardenberg RA, Gonzalez-Rivas D. Uniportal VATS right main bronchial sleeve resection (internal and external view). Asvide 2017;4:500. Available online: http://www. asvide.com/articles/1819 